\title{
Existence of a maximum balanced matching in the hypercube
}

\author{
Gyula O.H. Katona * \\ Rényi Institute \\ Budapest, Hungary \\ ohkatona@renyi.hu \\ Krisztián Tichler ${ }^{\dagger}$ \\ Eötvös University \\ Budapest, Hungary \\ ktichler@inf .elte.hu
}

June 27, 2012

\begin{abstract}
We prove, that for $n \neq 2$ the maximum possible $\left\lfloor 2^{n} / 2 n\right\rfloor$ edges can be chosen simultaneously from each parallel class of the $n$-cube in such a way, that no two edges have a common vertex.
\end{abstract}

\section{Introduction}

We consider the following problem for the $n$ dimensional hypercube. Select as many edges as possible from each parallel class simultaneously in such a way, that the set of edges form a matching of the hypercube. Here, matching is a subset of the edges, such that no two edges have a common vertex. More precisely, among all matchings of the hypercube maximize the minimum number of edges of the $n$ parallel classes of the edges. Obviously, no more than $\left\lfloor 2^{n} / 2 n\right\rfloor$ is possible, since each $n$ edges of a matching, one from each parallel class, need $2 n$ of the $2^{n}$ vertices of the hypercube. A matching is called a maximum balanced matching if it contains $\left\lfloor 2^{n} / 2 n\right\rfloor$ edges from each parallel class. Our main result is the following.

*The first author was supported by the Hungarian National Foundation for Scientific Research, grant number NK78439.

$\dagger$ This article is supported by the European Union and co-financed by the European Social Fund (ELTE TÁMOP-4.2.2/B-10/1-2010-0030). 
Theorem 1.1. There exists a maximum balanced matching of the $n$-cube for $n \neq 2$.

The problem emerged as a possible solution for a question of the authors ([2]) in combinatorial search theory.

There is a similar, well examined problem. List all words of length $n$ over the binary alphabet $\Sigma=\{0,1\}$ in such a way, that for each word the succeeding word differs only by a single bit, that is for each consecutive pair of words their Hamming distance is 1 . (The Hamming distance of words $u=t_{1} \cdots t_{n}$ and $v=t_{1}^{\prime} \cdots t_{n}^{\prime}$ over the alphabet $\Sigma$ is defined by $H(u, v)=$ $\left|\left\{i \in\{1, \ldots, n\} \mid t_{i} \neq t_{i}^{\prime}\right\}\right|$.) In other formulation, construct a Hamiltonian path (or cycle) in the $n$ dimensional hypercube.

One such Hamiltonian cycle for the $n$-cube is generated recursively from the Hamiltonian cycle for the $(n-1)$-cube. Take the same Hamiltonian path (eliminate an edge from the Hamiltonian cycle for $n-1$ ) in two parallel hyperplanes and add two edges, that connect their first and last vertices. This results in a Hamiltonian cycle for the $n$-cube. For the list of words this construction corresponds to the following recursive recipe: take two copies of the list for the words of length $n-1$, add a 0 prefix to each word in the first copy, reflect the order of the words in the second copy of the list and add a 1 prefix to each word, concatenate the two modified lists to get the list for word length $n$.

This list of words is called the binary-reflected Gray code. The name "Gray" refers to F. Gray, who patented this list of words as a solution to a communication problem involving digitization of analogue data ([3]).

More generally, any Hamiltonian path (cycle) in the $n$-cube is called a (cyclic) Gray code. There are many papers on Grey codes satisfying certain properties, for a survey see [1].

A long standing open problem on Gray codes was to construct a (cyclic) balanced one, i.e., one that contains a balanced number of edges from each of the $n$ parallel classes of edges. Since the number of edges in each parallel class must be even for a cyclic Gray code, the smallest possible positive difference is two. So for word lengths of non-2-powers, a balanced Grey code must have either the smallest even integer larger, or the largest even integer smaller than $2^{n} / n$ edges in each parallel class. Finally, G. S. Bhat and C. D. Savage ([4]) constructed a balanced Gray code for all $n$ using a proposed construction of J. Robinson and M. Cohn ([5]).

Note, that despite the similarity neither a balanced Grey code, nor a 
maximum balanced matching imply the existence of the other.

In section 2 we introduce some notations and prove our main lemma in proving Theorem 1.1. We complete its proof in section 3. In section 4 we introduce a generalization of the problem and prove some initial results in section 5. However, the problem remains open in general.

\section{Balanced cycle cover of the hypercube}

First, let us introduce some notations. Let $[n]=\{1, \ldots, n\}$ and $\left(\begin{array}{c}{[n]} \\ r\end{array}\right)=\{S \subseteq$ $[n]|| S \mid=r\}$. Furthermore let $\llbracket x \rrbracket_{r}=r\lfloor x / r\rfloor$. If $r=2$ we write shortly $\llbracket x \rrbracket$ instead of $\llbracket x \rrbracket_{2}$. If $\Sigma$ is an alphabet let $\Sigma^{n}$ denote the set of words of length $n$ over $\Sigma$.

Let $B_{n}$ be the $n$ dimensional hypercube, $B_{n}=\left\langle V\left(B_{n}\right), \mathcal{E}\left(B_{n}\right)\right\rangle$, where $V\left(B_{n}\right)=$ $\{0,1\}^{n}$ is the set of binary words of length $n$ and $\mathcal{E}\left(B_{n}\right)=\{\{u, v\} \mid H(u, v)=$ $1\}$.

$\mathcal{E}=\mathcal{E}\left(B_{n}\right)$ has a natural decomposition $\mathcal{E}=\cup_{i=1}^{n} \mathcal{E}_{i}$ according to the directions, formally

$$
\left\{b_{1} \cdots b_{n}, b_{1}^{\prime} \cdots b_{n}^{\prime}\right\} \in \mathcal{E}_{i} \text { if and only if } b_{j}=b_{j}^{\prime}, j \neq i \text { and } b_{i} \neq b_{i}^{\prime} .
$$

For $\mathcal{E}^{\prime} \subseteq \mathcal{E}$ and $i \in[n]$ let

$$
\lambda_{i}=\lambda_{i}\left(\mathcal{E}^{\prime}\right)=\left|\mathcal{E}^{\prime} \cap \mathcal{E}_{i}\right|
$$

furthermore let

$$
\chi\left(\mathcal{E}^{\prime}\right)=\left(\lambda_{1}, \ldots, \lambda_{n}\right)
$$

be the profile vector of $\mathcal{E}^{\prime}$.

For a subgraph $G=\langle V, \mathcal{E}\rangle$ of $B_{n}$ and $b \in\{0,1\}$ let

$$
G^{b}=\left\langle\{v b \mid v \in V\},\left\{\left\{v_{1} b, v_{2} b\right\} \mid\left\{v_{1}, v_{2}\right\} \in \mathcal{E}\right\}\right\rangle .
$$

If $\mathcal{G}=\left\{G_{1}, \ldots, G_{k}\right\}$, then let $\mathcal{G}^{b}=\left\{G_{1}^{b}, \ldots, G_{k}^{b}\right\}$ and $\mathcal{E}(\mathcal{G})=\bigcup_{i=1}^{k} \mathcal{E}\left(G_{i}\right)$.

For $v=b_{1} \cdots b_{n} \in V\left(B_{n}\right)$ let

$$
\sigma_{i}(v)=b_{1} \cdots b_{i-1} \bar{b}_{i} b_{i+1} \cdots b_{n} \quad(\bar{b}=1-b) .
$$

If $E \in \mathcal{E}\left(B_{n}\right)$ let

$$
\sigma_{i}(E)=\left\{\begin{array}{ll}
\left\{\sigma_{i}(u), \sigma_{i}(v)\right\} & \text { if }\{u, v\} \notin \mathcal{E}_{i} \\
\{u, v\} & \text { if }\{u, v\} \in \mathcal{E}_{i}
\end{array} .\right.
$$


Let us introduce the notations $\sigma_{i}\left(V^{\prime}\right)=\left\{\sigma_{i}(v) \mid v \in V^{\prime}\right\}$ for $V^{\prime} \subseteq V\left(B_{n}\right)$ and $\sigma_{i}\left(\mathcal{E}^{\prime}\right)=\left\{\sigma_{i}(E) \mid E \in \mathcal{E}^{\prime}\right\}$ for $\mathcal{E}^{\prime} \subseteq \mathcal{E}\left(B_{n}\right)$. Given a subgraph $G=\langle V, \mathcal{E}\rangle$ of $B_{n}$ let $\sigma_{i}(G)=\left\langle\sigma_{i}(V), \sigma_{i}(\mathcal{E})\right\rangle$. So $\sigma_{i}$ gives nothing else, but the mirror image w.r.t. direction $i$.

We know ([4]) that, there exists a balanced Grey code. On one hand, the following lemma states less, the existence of a balanced cover of cycles instead of a single balanced Hamiltonian cycle. On the other hand, the lemma gives us a small, specific cycle, containing edges in all direction, that will be used for correcting a later specified almost balanced matching.

Lemma 2.1. For $n \geq 3$ there exist a set of cycles $\mathcal{C}_{n}=\left\{C_{0}, C_{1}, \ldots, C_{t}\right\}$ of $B_{n}$ for some $t=t(n)$ having the following properties.

(i) $\bigcup_{i=0}^{t} V\left(C_{i}\right)=V\left(B_{n}\right)$,

(ii) $V\left(C_{i}\right) \cap V\left(C_{j}\right)=\emptyset(i \neq j ; 0 \leq i, j \leq t)$,

(iii) $C_{0}=\left(v_{1}, E_{1}, \ldots, v_{2 n}, E_{2 n}\right), E_{i}=\left\{v_{i}, v_{i(\bmod 2 n)+1}\right\}(i \in[2 n])$, $E_{i}, E_{2 n-i} \in \mathcal{E}_{i},(i \in[n-1]), E_{n}, E_{2 n} \in \mathcal{E}_{n}$,

(iv) let $\lambda_{i}=\lambda_{i}\left(\mathcal{E}\left(\mathcal{C}_{n}\right)\right)$, then $\left|\lambda_{i}-\lambda_{j}\right| \leq 2(1 \leq i, j \leq n)$.

A set of cycles satisfying $(i)-(i v)$ is called a balanced cycle cover (bcc).

Note, that since $B_{n}$ is a bipartite graph, it has only even cycles so the value of $\lambda_{j}$ is even as well $(1 \leq j \leq n)$. Furthermore, $\lambda_{j}\left(\mathcal{E}\left(C_{i}\right)\right)$ is even, too, for $0 \leq i \leq t, 1 \leq j \leq n$.

Circuits of the form $\left(v_{1}, E, v_{2}, E\right), v_{1}, v_{2} \in V\left(B_{n}\right), E=\left\{v_{1}, v_{2}\right\}, E \in$ $\mathcal{E}\left(B_{n}\right)$ are considered to be cycles, as well.

Proof of Lemma 2.1. The proof is by induction. It is easy to construct a bcc for $n=3$ or $n=4$. Suppose that we have a bcc for $B_{n}$ and let us construct one for $B_{n+1}$.

The edges of $\mathcal{E}_{n+1}$ connect two disjoint copies of $B_{n}$ in $B_{n+1}$ since $\mathcal{E}_{n+1}=$ $\left\{\{u 0, u 1\} \mid u \in\{0,1\}^{n}\right\}$. By the induction hypothesis there exist a bcc $\mathcal{C}_{n}=$ $\left\{C_{0}, \ldots, C_{t}\right\}$ in $B_{n}$, so that it has a profile

$$
\chi\left(\mathcal{E}\left(\mathcal{C}_{n}\right)\right)=\left(\lambda_{1}, \ldots, \lambda_{n}\right),
$$

where $\lambda_{1}=\cdots=\lambda_{s}, \lambda_{s+1}=\cdots \lambda_{n}, \lambda_{s+1}=\lambda_{s}+2$, for some $s \in[n]$ and all $\lambda_{i}$ 's are even. 
Then let $\mathcal{C}$ be the following cover of $V\left(B_{n+1}\right)$ by vertex disjoint cycles $\mathcal{C}=\mathcal{C}_{n}^{0} \cup \mathcal{C}_{n}^{1}=\left\{C_{0}^{0}, \ldots, C_{t}^{0}, C_{0}^{1} \ldots, C_{t}^{1}\right\}$. So $C_{0}^{b}=\left\{v_{1} b, E_{1}^{b}, \ldots, v_{2 n} b, E_{2 n}^{b}\right\}$, where $E_{i}^{b}=\left\{v_{i} b, v_{i(\bmod 2 n)+1} b\right\}(b \in\{0,1\})$. By the induction hypothesis $E_{i}^{b}, E_{2 n-i}^{b} \in \mathcal{E}_{i}, E_{n}^{b}, E_{2 n}^{b} \in \mathcal{E}_{n}(i \in[n-1], b \in\{0,1\})$.

Observe, that $\mathcal{C}$ has the property

$$
C \in \mathcal{C} \Leftrightarrow \sigma_{n+1}(C) \in \mathcal{C}
$$

SO

$$
E \in \mathcal{E}(\mathcal{C}) \Leftrightarrow \sigma_{n+1}(E) \in \mathcal{E}(\mathcal{C})
$$

holds as well.

$\mathcal{C}$ has properties (i)-(ii), but does not satisfy properties (iii)-(iv). We have

$$
\chi(\mathcal{E}(\mathcal{C}))=\left(2 \lambda_{1}, \ldots, 2 \lambda_{n}, 0\right) .
$$

Replace $C_{0}^{0}$ and $C_{0}^{1}$ by two other cycles. Let the set of their edges be

$$
\left\{E_{1}^{0}, \ldots, E_{n}^{0},\left\{v_{n+1} 0, v_{n+1} 1\right\}, E_{n}^{1}, \ldots, E_{1}^{1},\left\{v_{1} 0, v_{1} 1\right\}\right\}
$$

and

$$
\left\{E_{n+2}^{0}, \ldots, E_{2 n-1}^{0},\left\{v_{2 n} 0, v_{2 n} 1\right\}, E_{2 n-1}^{1}, \ldots, E_{n+2}^{1},\left\{v_{n+2} 0, v_{n+2} 1\right\}\right\} .
$$

By renaming the cycles we get a set of vertex disjoint cycles $\left\{C_{0}, \ldots, C_{2 t+1}\right\}$ covering $V\left(B_{n+1}\right)$, where $\mathcal{E}\left(C_{0}\right)$ equals (3). We use the same notation $\mathcal{C}$ for the new cycle system. Note, that $\mathcal{C}$ satisfies (i)-(iii) and (1). Furthermore,

$$
\chi(\mathcal{E}(\mathcal{C}))=\left(2 \lambda_{1}, \ldots, 2 \lambda_{n-2}, 2 \lambda_{n-1}-2,2 \lambda_{n}-2,4\right) .
$$

The first $n$ components of the profile vector differ by maximum 2 and are at least 4 for $n \geq 4$. Take an edge $E \in \mathcal{E}\left(\mathcal{C} \backslash\left\{C_{0}\right\}\right)$ of $\mathcal{E}_{i}(i \in[n])$, where $\lambda_{i}(\mathcal{E}(\mathcal{C}))$ is at least as large as any other component. W.l.o.g. suppose, that $E=\{u 0, v 0\}\left(u, v \in\{0,1\}^{n}\right)$. Then $E^{\prime}=\sigma_{n+1}(E)=\{u 1, v 1\} \in \mathcal{E}(\mathcal{C})$ holds as well by (2). Replace $E$ and $E^{\prime}$ by $E^{\prime \prime}=\{u 0, u 1\}$ and $E^{\prime \prime \prime}=\{v 0, v 1\}$ (see Figure 1). This transformation decreases $\lambda_{i}(\mathcal{E}(\mathcal{C}))$ by 2 and increases $\lambda_{n+1}(\mathcal{E}(\mathcal{C}))$ by 2 , while properties (i)-(iii) still hold.

Observe, that if $E$ and $E^{\prime}$ belong to different cycles

$$
C_{1}=\left(w_{0}, E_{0}, \ldots, w_{k}, E_{k}\right) \text { and } C_{2}=\sigma_{n+1}\left(C_{1}\right)=\left(w_{0}^{\prime}, E_{0}^{\prime}, \ldots, w_{k}^{\prime}, E_{k}^{\prime}\right)
$$




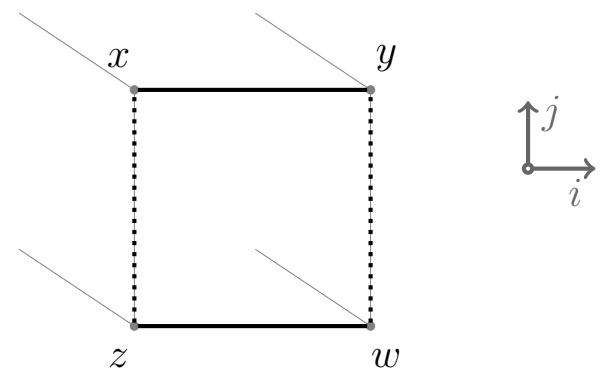

Figure 1: The following basic transformation is used many times. Suppose, that $\{x, y\},\{z, w\} \in \mathcal{E}$ and both have color (direction) $i$, suppose furthermore, that $\{x, z\},\{y, w\} \notin \mathcal{E}$ and both have color (direction) $j$, then flipping the pairs of edges decreases $\lambda_{i}$ by 2 and increases $\lambda_{j}$ by 2 .

where $k \geq 1, w_{0}=u 0, w_{1}=v 0, E_{0}=E, E_{0}^{\prime}=E^{\prime}, w_{i}^{\prime}=\sigma_{n+1}\left(w_{i}\right)(0 \leq i \leq k)$, then $C_{1}$ and $C_{2}$ is replaced by a single, larger cycle

$$
C=\left(w_{1}, E_{1}, \ldots, w_{k}, E_{k}, w_{0}, E^{\prime \prime}, w_{0}^{\prime}, E_{k}^{\prime}, w_{k}^{\prime}, \ldots, E_{1}^{\prime}, w_{1}^{\prime}, E^{\prime \prime \prime}\right) .
$$

On the other hand if $E$ and $E^{\prime}$ are edges of the same cycle

$$
C=\left(w_{0}, E_{0}, \ldots, w_{k}, E_{k}\right)
$$

satisfying $\sigma_{n+1}(C)=C$, where $k \geq 3, E_{0}=E, E_{t}=E^{\prime}$ (for some $2 \leq t \leq$ $k-1), w_{0}=u 0, w_{1}=v 0, w_{t}=v 1, w_{t+1}=u 1$, than $C$ is replaced by two smaller cycles

$C_{1}=\left(w_{1}, E_{1}, \ldots, w_{t-1}, E_{t-1}, w_{t}, E^{\prime \prime \prime}\right)$ and $C_{2}=\left(w_{t+1}, E_{t+1}, \ldots, w_{k}, E_{k}, w_{0}, E^{\prime \prime}\right)$.

Easy to check, that in both cases also (1) holds for the modified family of cycles. We use the same notation $\mathcal{C}$ for for the new cycle system.

Repeat the previous step until the cycle cover becomes balanced. We can do this, since the preconditions of the transformation (properties (i)-(iii) and (1)) still hold after each execution.

We also need, that there is at least one pair of edges not belonging to $C_{0}$ to flip. But this is true, since

$$
\left|\mathcal{E}\left(C_{0}\right)\right|+\lambda_{n+1}(\mathcal{E}(\mathcal{C})) \leq 2 n+2+\frac{2^{n+1}}{n+1}<2^{n+1}=|\mathcal{E}(\mathcal{C})| \quad(n \geq 4) .
$$

For that actual $\mathcal{C}$ let $\mathcal{C}_{n+1}=\mathcal{C}$. Properties (i)-(iv) hold for $\mathcal{C}_{n+1}$. 


\section{Maximum balanced matching in the hyper- cube}

\subsection{Case of $n \leq 7$}

For $n=1$ and $n=2$ the statement is obvious. For $n=3$ a possible solution is to take the even edges of the following Grey code (Hamiltonian path) $G(3)=v_{0}, v_{1}, \ldots, v_{7}$.

$$
\begin{aligned}
& v_{0}=000 \quad v_{2}=011 \quad v_{4}=101 \quad v_{6}=110 \\
& v_{1}=010 \quad v_{3}=001 \quad v_{5}=100 \quad v_{7}=111 \\
& \begin{array}{llllllll}
v_{0} & v_{1} & v_{2} & v_{3} & v_{4} & v_{5} & v_{6} & v_{7}
\end{array} \\
& \begin{array}{lllllll}
2 & \underline{3} & 2 & \underline{1} & 3 & \underline{\mathbf{2}} & 1
\end{array}
\end{aligned}
$$

For $n=4$ consider the following cyclic Grey code (Hamiltonian cycle) $G(4)=v_{0}, v_{1}, \ldots, v_{15}$.

$$
\begin{aligned}
& v_{0}=0000 \quad v_{4}=0110 \quad v_{8}=1111 \quad v_{12}=1001 \\
& v_{1}=1000 \quad v_{5}=0100 \quad v_{9}=0111 \quad v_{13}=1011 \\
& v_{2}=1010 \quad v_{6}=1100 \quad v_{10}=0101 \quad v_{14}=0011 \\
& v_{3}=1110 \quad v_{7}=1101 \quad v_{11}=0001 \quad v_{15}=0010 \\
& \begin{array}{llllllllllllllll}
v_{0} & v_{1} & v_{2} & v_{3} & v_{4} & v_{5} & v_{6} & v_{7} & v_{8} & v_{9} & v_{10} & v_{11} & v_{12} & v_{13} & v_{14} & v_{15}
\end{array} \\
& \begin{array}{llllllllllllllll}
\underline{\mathbf{1}} & 3 & \underline{\mathbf{2}} & 1 & \underline{\mathbf{3}} & 1 & \underline{\mathbf{4}} & 3 & \underline{\mathbf{1}} & 3 & \underline{\mathbf{2}} & 1 & \underline{\mathbf{3}} & 1 & \underline{\mathbf{4}} & 3
\end{array}
\end{aligned}
$$

$G(4)$ have some interesting properties, we shall need the following later:

$$
\left\{v_{2 s}, v_{2 s+1}\right\} \in \mathcal{E}_{s(\bmod 4)+1} \quad(s=0, \ldots, 7) .
$$

So the odd edges give a maximum balanced matching, $\mathcal{M}_{4}$ for $n=4$.

For $n=5,6,7$ we consider $B_{n}$ as $B_{2} \times B_{3}, B_{3} \times B_{3}$ and $B_{3} \times B_{4}$, respectively. We use only the edges of the above Grey codes $G(3)$ and $G(4)$ in the corresponding subcubes to construct maximum balanced matchings $\mathcal{M}_{5}, \mathcal{M}_{6}, \mathcal{M}_{7}$. One possible solution for each $n \in\{5,6,7\}$ can be seen on Figure 2.

\subsection{Case of $n \geq 4, n$ is a power of 2}

For $n \geq 4, n$ is a power of 2 , we can construct a complete matching with equal number of edges in each parallel class. We construct recursively a cyclic Grey 

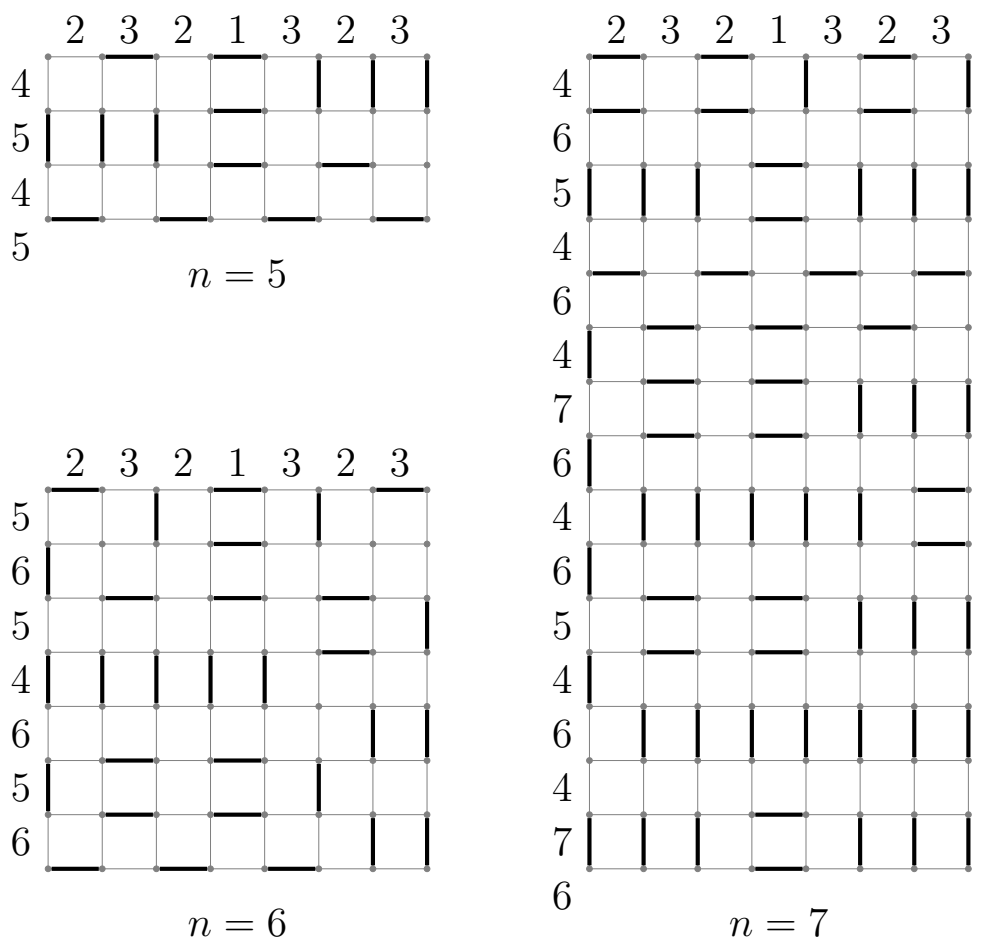

Figure 2: Maximum balanced matchings for $n=5,6,7$. The parallel classes $\mathcal{E}_{i}(1 \leq i \leq 7)$ are denoted shortly by $1,2,3,4,5,6,7$.

code $G\left(2^{t}\right)$ of $B_{2^{t}},(t \geq 2)$, such that its odd edges form the desired complete matching. Furthermore, the Grey code will have the following property:

the $i$ th and the $\left(i+2^{2^{t}-1}\right)$ th element belong

to the same parallel class $\left(1 \leq i \leq 2^{2^{t}-1}\right)$.

For $n=4$ we have already constructed a cyclic Grey code. By (4) it has property (5). Suppose, that we have already constructed a Grey code $G\left(2^{t}\right)=$ $v_{1}, \ldots, v_{2^{2}}$ satisfying (5). We construct a Grey code satisfying (5) for $B_{2^{t+1}}=$ $B_{2^{t}} \times B_{2^{t}}$. By the induction hypothesis, the following Hamiltonian cycle is 


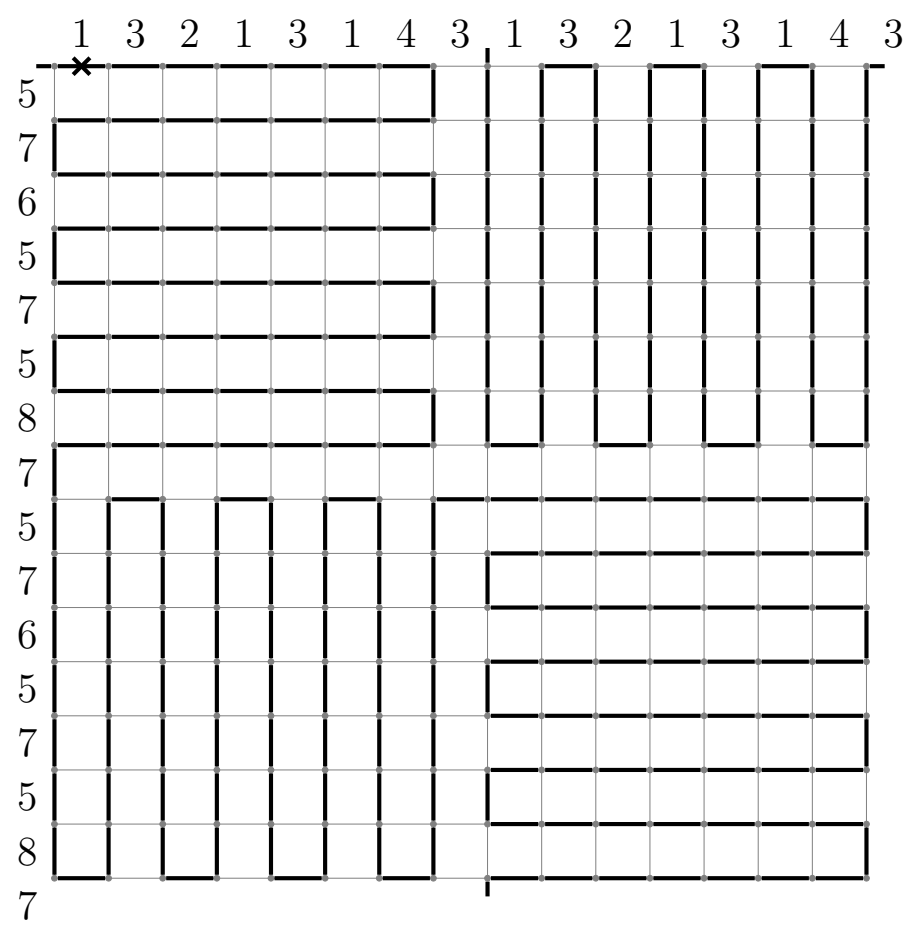

Figure 3: Construction of a cyclic Grey code for the power of $2(n=8)$. Taking every second edge from the marked one yields a maximum balanced matching.

appropriate (for $n=8$, see Figure 3).

$$
\begin{array}{r}
G\left(2^{t+1}\right)=\left(v_{1}, v_{1}\right),\left(v_{1}, v_{2}\right), \ldots,\left(v_{1}, v_{2^{2^{t}-1}}\right),\left(v_{2}, v_{2^{2^{t}-1}}\right), \ldots,\left(v_{2}, v_{1}\right),\left(v_{3}, v_{1}\right), \\
\ldots,\left(v_{3}, v_{2^{2^{t}-1}}\right),\left(v_{4}, v_{2^{2^{t}-1}}\right), \ldots, \ldots,\left(v_{2^{2^{t}-1}}, v_{1}\right),\left(v_{2^{2^{t}-1}+1}, v_{1}\right),\left(v_{2^{2^{t}-1}+2}, v_{1}\right), \\
\ldots,\left(v_{2^{2^{t}}}, v_{1}\right),\left(v_{2^{2^{t}}}, v_{2}\right), \ldots,\left(v_{2^{2^{t}-1}+1}, v_{2}\right),\left(v_{2^{2^{t}-1}+1}, v_{3}\right), \ldots, \\
\ldots,\left(v_{2^{2^{t}-1}+1}, v_{2^{2^{t}-1}}\right),\left(v_{2^{2^{t}-1}+1}, v_{2^{2^{t}-1}+1}\right),\left(v_{2^{2^{t}-1}+1}, v_{2^{2^{t}-1}+2}\right), \ldots, \\
\left(v_{2^{2^{t}-1}+1}, v_{2^{2^{t}}}\right),\left(v_{2^{2^{t}-1}+2}, v_{2^{2^{t}}}\right), \ldots,\left(v_{2^{2^{t}-1}+2}, v_{2^{2^{t}-1}+1}\right),\left(v_{2^{2^{t}-1}+3}, v_{2^{2^{t}-1}+1}\right), \\
\ldots, \ldots,\left(v_{2^{2^{t}}}, v_{2^{2^{t}-1}+1}\right),\left(v_{1}, v_{2^{2^{t}-1}+1}\right), \ldots,\left(v_{2^{2^{t}-1}}, v_{2^{2^{t}-1}+1}\right),\left(v_{2^{2^{t}-1}}, v_{2^{2^{t}-1}+2}\right), \\
\ldots,\left(v_{1}, v_{2^{2^{t}-1}+2}\right),\left(v_{1}, v_{2^{2^{t}-1}+3}\right), \ldots, \ldots,\left(v_{1}, v_{2^{2^{t}}}\right) .
\end{array}
$$




\subsection{Case of $n \geq 9, n$ is not a power of 2}

For $n \geq 9, n$ is not a power of 2 , we construct a maximum balanced matching using a balanced cycle cover of Lemma 2.1 for $B_{n-4}$. Note, that in this case $2^{n}-2 n\left\lfloor 2^{n} / 2 n\right\rfloor \geq 2$ holds, so we can afford not to cover at least 2 vertices.

$B_{n}=B_{n-4} \times B_{4}$, so we can assume that the vertices of $B_{n}$ are of the form $\left(u_{i}, v_{j}\right), 1 \leq i \leq 2^{n-4}, 0 \leq j \leq 15$, where $G(4)=v_{0}, \ldots, v_{15}$. Let $\mathcal{C}_{n-4}=\left\{C_{0}, C_{1}, \ldots, C_{t}\right\}$ be a balanced cycle cover of $B_{n-4}$, such that

$$
\bigcup_{i=0}^{t} V\left(C_{i}\right)=\left\{u_{1}, \ldots, u_{2^{n-4}}\right\}
$$

and

$$
\begin{gathered}
\mathcal{E}\left(C_{0}\right)=\left\{\left\{u_{1}, u_{2}\right\},\left\{u_{2}, u_{3}\right\}, \ldots,\left\{u_{2 n-9}, u_{2 n-8}\right\},\left\{u_{2 n-8}, u_{1}\right\}\right\}, \\
\left\{u_{i}, u_{i+1}\right\},\left\{u_{2 n-8-i}, u_{2 n-7-i}\right\} \in \mathcal{E}_{i}(1 \leq i \leq n-5), \\
\left\{u_{n-4}, u_{n-3}\right\},\left\{u_{2 n-8}, u_{1}\right\} \in \mathcal{E}_{n-4} .
\end{gathered}
$$

By (4) we have

$$
\begin{aligned}
\left\{\left(u_{i}, v_{2 j}\right),\left(u_{i}, v_{2 j+1}\right)\right\},\left\{\left(u_{i}, v_{2 j+8}\right),\left(u_{i}, v_{2 j+9}\right)\right\} & \in \mathcal{E}_{n-3+j} \\
& j=0,1,2,3, \quad 1 \leq i \leq 2^{n-4}
\end{aligned}
$$

Let $\mathcal{M}$ be the following matching. If $E=\left\{u_{i}, u_{j}\right\}$ is an odd edge of $C_{i}(i \geq 1)$, then let

$$
\left\{\left(u_{i}, v_{0}\right),\left(u_{j}, v_{0}\right)\right\}, \ldots,\left\{\left(u_{i}, v_{7}\right),\left(u_{j}, v_{7}\right)\right\} \in \mathcal{M}
$$

otherwise let

$$
\left\{\left(u_{i}, v_{8}\right),\left(u_{j}, v_{8}\right)\right\}, \ldots,\left\{\left(u_{i}, v_{15}\right),\left(u_{j}, v_{15}\right)\right\} \in \mathcal{M} .
$$

If $E$ is an odd edge of $C_{0}$, then let

$$
\left\{\left(u_{i}, v_{1}\right),\left(u_{j}, v_{1}\right)\right\},\left\{\left(u_{i}, v_{3}\right),\left(u_{j}, v_{3}\right)\right\}, \ldots,\left\{\left(u_{i}, v_{15}\right),\left(u_{j}, v_{15}\right)\right\} \in \mathcal{M}
$$

otherwise let

$$
\left\{\left(u_{i}, v_{0}\right),\left(u_{j}, v_{0}\right)\right\},\left\{\left(u_{i}, v_{2}\right),\left(u_{j}, v_{2}\right)\right\}, \ldots,\left\{\left(u_{i}, v_{14}\right),\left(u_{j}, v_{14}\right)\right\} \in \mathcal{M}
$$

These edges are called $C_{0}$-edges. 
If $\mathcal{C}_{n-4}$ has a profile $\left(\lambda_{1}^{\prime}, \ldots, \lambda_{n-4}^{\prime}\right), \lambda_{1}^{\prime}=\cdots=\lambda_{s}^{\prime}, \lambda_{s+1}^{\prime}=\cdots=\lambda_{n-4}^{\prime}, \lambda_{s+1}^{\prime}=$ $\lambda_{s}^{\prime}+2$, for some $1 \leq s<n-4$, then we have

$$
\chi(\mathcal{M})=\left(8 \lambda_{1}^{\prime}, \ldots, 8 \lambda_{n-4}^{\prime}, 0,0,0,0\right) .
$$

Take 2 edges $E=\left\{\left(u_{i}, v_{j}\right),\left(u_{i^{\prime}}, v_{j}\right)\right\}$ and $E^{\prime}=\left\{\left(u_{i}, v_{j+1}\right),\left(u_{i^{\prime}}, v_{j+1}\right)\right\}$, such that $j$ is even and $\left\{u_{i}, u_{i^{\prime}}\right\} \in \mathcal{E}\left(\mathcal{C}_{n-4} \backslash\left\{C_{0}\right\}\right)$. Remove $E$ and $E^{\prime}$ from $\mathcal{M}$ and add $\left\{\left(u_{i}, v_{j}\right),\left(u_{i}, v_{j+1}\right)\right\}$ and $\left\{\left(u_{i^{\prime}}, v_{j}\right),\left(u_{i^{\prime}}, v_{j+1}\right)\right\}$. So if $E, E^{\prime} \in \mathcal{M} \cap \mathcal{E}_{k}$, then we are decreased $\lambda_{k}(\mathcal{M})$ by 2 , while increased one of the last 4 components of $\chi(\mathcal{M})$ by 2 (by (4)).

Repeating the above transformation in an approriate order, we can reach, that all components of $\chi(\mathcal{M})$ differ by either 0 or 2 if there are enough edges initially in $\mathcal{E}\left(\mathcal{C}_{n-4} \backslash\left\{C_{0}\right\}\right) \cap \mathcal{E}_{k}(1 \leq k \leq n-4)$.

In the initial matching there are at least $8 \llbracket 2^{n-4} /(n-4) \rrbracket-16$ edges in $\mathcal{E}\left(\mathcal{C}_{n-4} \backslash\left\{C_{0}\right\}\right)$ in each parallel class, while at most $\llbracket 2^{n} / 2 n \rrbracket$ needed. Substituting $n=9$ the first quantity is larger than the second one. For $n \geq 10$ we have

$$
8 \llbracket \frac{2^{n-4}}{n-4} \rrbracket-16 \geq 8\left(\frac{2^{n-4}}{n-4}-2\right)-16 \geq \frac{2^{n}}{2 n} \geq \llbracket \frac{2^{n}}{2 n} \rrbracket
$$

The middle inequality is equivalent to the inequality $2^{n-4} \geq n(n-4)$, which holds for $n \geq 10$.

So we have a matching $\mathcal{M}$, such that

$$
\chi(\mathcal{M})=\left(\lambda_{1}, \ldots, \lambda_{n}\right),
$$

where $\lambda_{i_{1}}=\cdots=\lambda_{i_{s}}, \lambda_{i_{s+1}}=\cdots=\lambda_{i_{n}}, \lambda_{i_{s+1}}=\lambda_{i_{s}}+2$ with $2(n-s)=$ $2^{n}-2 n\left\lfloor 2^{n} / 2 n\right\rfloor$ and all $\lambda_{i_{j}}$ 's are even.

Note, that we can set $\left\{i_{1}, \ldots, i_{s}\right\}$ to be any specific $s$-subset of $[n]$ and $\mathcal{M}$ still contains all $C_{0^{-}}$-edges. $\lambda_{i_{1}}$ equals either $\left\lfloor 2^{n} / 2 n\right\rfloor-1$ or $\left\lfloor 2^{n} / 2 n\right\rfloor$. If $\lambda_{i_{1}}=\left\lfloor 2^{n} / 2 n\right\rfloor-1$ then the $C_{0}$-edges will be used for correction. We distinguish 5 cases (Figure 4).

Case 1. If $s \geq n / 2$, then we are either ready, since $\lambda_{i_{1}}=\left\lfloor 2^{n} / 2 n\right\rfloor$ (if $s>n / 2$ ) or $n$ is a power of 2 (if $s=n / 2$ ), since $2^{n} / 2 n=\left\lfloor 2^{n} / 2 n\right\rfloor$ can not hold otherwise. The case of $n$ is a power of 2 is already discussed.

Case 2. Let $s<(n-4) / 2$. Assume, that $\left\lfloor 2^{n} / 2 n\right\rfloor-1=\lambda_{1}=\lambda_{3}=\cdots=$ 


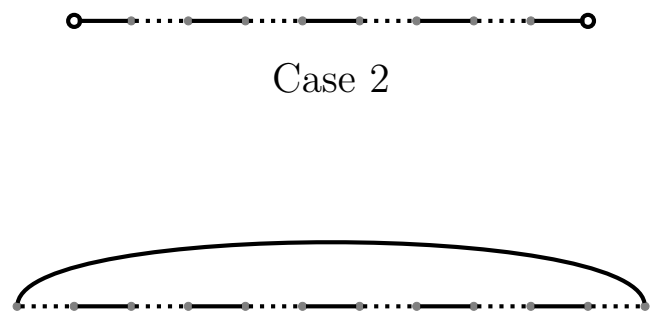

Case 3

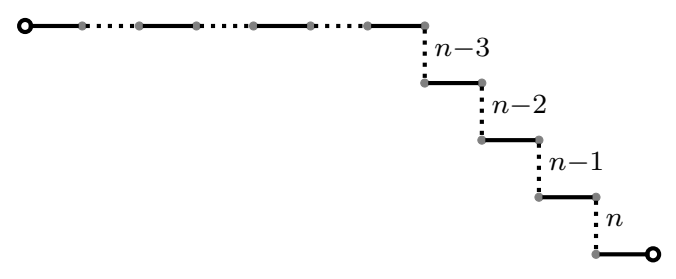

Cases 4 and 5

Figure 4: Balanceness correction using the $C_{0}$-edges. (The original edges are replaced by the dotted ones.)

$\lambda_{2 s-1}$. Let us introduce the notation

$$
\begin{aligned}
\mathcal{D}_{k, s}=\left\{\left\{\left(u_{k}, v_{0}\right)\right.\right. & \left.,\left(u_{k(\bmod (2 n-8))+1}, v_{0}\right)\right\} \\
\left\{\left(u_{k+1}(\bmod (2 n-8))+1\right.\right. & \left.\left.v_{0}\right),\left(u_{k+2(\bmod (2 n-8))+1}, v_{0}\right)\right\}, \\
& \left.\quad \ldots,\left\{\left(u_{k+2 s-3(\bmod (2 n-8))+1}, v_{0}\right),\left(u_{k+2 s-2(\bmod (2 n-8))+1}, v_{0}\right)\right\}\right\} .
\end{aligned}
$$

By (6), (7) and (8) $\mathcal{M} \backslash \mathcal{D}_{2 n-8, s+1} \cup \mathcal{D}_{1, s}$ is a maximum balanced matching, since the $\lambda_{2 i}$ 's are decreased for $i=n-4$ and $i \in[s]$, while the $\lambda_{2 i-1}$ 's are increased by 1 , for $i \in[s]$.

Case 3. If $s=(n-4) / 2$ and $\left\lfloor 2^{n} / 2 n\right\rfloor-1=\lambda_{1}=\lambda_{3}=\cdots=\lambda_{n-5}$ then $\mathcal{M} \backslash \mathcal{D}_{2,(n-4) / 2} \cup \mathcal{D}_{1,(n-4) / 2}$ is a maximum balanced matching.

Case 4. $s=\lfloor(n-4) / 2\rfloor+1$. We can assume, that $\left\lfloor 2^{n} / 2 n\right\rfloor-1=\lambda_{3}=$ $\cdots=\lambda_{2\lfloor(n-4) / 2\rfloor-5}=\lambda_{n-3}=\lambda_{n-2}=\lambda_{n-1}=\lambda_{n}$, while all other components of $\chi(\mathcal{M})$ equal to $\left\lfloor 2^{n} / 2 n\right\rfloor+1$. Let

$$
\begin{aligned}
\mathcal{D}_{4}^{-}=\left\{\left\{\left(u_{2\lfloor(n-4) / 2\rfloor-3}, v_{1}\right),\left(u_{2\lfloor(n-4) / 2\rfloor-2}, v_{1}\right)\right\},\right. & \\
\left\{\left(u_{2\lfloor(n-4) / 2\rfloor-2}, v_{2}\right),\left(u_{2\lfloor(n-4) / 2\rfloor-1}, v_{2}\right)\right\} & ,\left\{\left(u_{2\lfloor(n-4) / 2\rfloor-1}, v_{3}\right),\left(u_{2\lfloor(n-4) / 2\rfloor}, v_{3}\right)\right\}, \\
& \left.\left\{\left(u_{2\lfloor(n-4) / 2\rfloor}, v_{8}\right),\left(u_{2\lfloor(n-4) / 2\rfloor+1}, v_{8}\right)\right\}\right\}
\end{aligned}
$$

and

$$
\begin{aligned}
\mathcal{D}_{4}^{+}=\left\{\left\{\left(u_{2\lfloor(n-4) / 2\rfloor-3}, v_{0}\right),\left(u_{2\lfloor(n-4) / 2\rfloor-3}, v_{1}\right)\right\},\right. & \\
\left\{\left(u_{2\lfloor(n-4) / 2\rfloor-2}, v_{1}\right),\left(u_{2\lfloor(n-4) / 2\rfloor-2}, v_{2}\right)\right\}, & \left\{\left(u_{2\lfloor(n-4) / 2\rfloor-1}, v_{2}\right),\left(u_{2\lfloor(n-4) / 2\rfloor-1}, v_{3}\right)\right\}, \\
& \left.\left\{\left(u_{2\lfloor(n-4) / 2\rfloor}, v_{3}\right),\left(u_{2\lfloor(n-4) / 2\rfloor}, v_{8}\right)\right\}\right\} .
\end{aligned}
$$


Note, that in $G(4)\left\{v_{0}, v_{1}\right\},\left\{v_{1}, v_{2}\right\},\left\{v_{2}, v_{3}\right\},\left\{v_{3}, v_{8}\right\}$ belong to 4 different classes of edges. $\mathcal{M} \backslash\left(\mathcal{D}_{2, s-3} \cup \mathcal{D}_{4}^{-}\right) \cup \mathcal{D}_{3, s-4} \cup \mathcal{D}_{4}^{+}$is a maximum balanced matching, since the $\lambda_{2 i}$ 's for $1 \leq i \leq\lfloor(n-4) / 2\rfloor-2$ and the $\lambda_{i}$ 's for $2\lfloor(n-4) / 2\rfloor-3 \leq i \leq 2\lfloor(n-4) / 2\rfloor$ are decreased, while the $\lambda_{2 i-1}$ 's for $2 \leq i \leq\lfloor(n-4) / 2\rfloor-2$ and the $\lambda_{i}$ 's for $n-3 \leq i \leq n$ are increased by 1 .

Case 5. $s=\lfloor(n-4) / 2\rfloor+2$ and $n$ is odd. (Note, that the case of even $n$ was already considered in Case 1.) We can assume, that $\left\lfloor 2^{n} / 2 n\right\rfloor-1=$ $\lambda_{1}=\lambda_{3}=\cdots=\lambda_{2\lfloor(n-4) / 2\rfloor-5}=\lambda_{n-3}=\lambda_{n-2}=\lambda_{n-1}=\lambda_{n}$, while all other components of $\chi(\mathcal{M})$ equal to $\left\lfloor 2^{n} / 2 n\right\rfloor+1$.

$\mathcal{M} \backslash\left(\mathcal{D}_{2 n-8, s-3} \cup \mathcal{D}_{4}^{-}\right) \cup \mathcal{D}_{1, s-4} \cup \mathcal{D}_{4}^{+}$is a maximum balanced matching, since $\lambda_{n-4}$, the $\lambda_{2 i}$ 's for $1 \leq i \leq\lfloor(n-4) / 2\rfloor-2$ and the $\lambda_{i}$ 's for $2\lfloor(n-4) / 2\rfloor-3 \leq$ $i \leq 2\lfloor(n-4) / 2\rfloor$ are decreased, while the $\lambda_{2 i-1}$ 's for $1 \leq i \leq\lfloor(n-4) / 2\rfloor-2$ and the $\lambda_{i}$ 's for $n-3 \leq i \leq n$ are increased by 1 . (Note, that we have $n-4 \neq 2\lfloor(n-4) / 2\rfloor$ in this case. $)$

We could achieve in all the 5 cases, that each of the parallel classes contain at least $\left\lfloor 2^{n} / 2 n\right\rfloor$ elements.

\section{Balanceness of hypergraphs}

Let us consider the following generalization of our problem. Let $\mathcal{H}=\langle V, \mathcal{E}\rangle$ be a hypergraph (i.e., $\mathcal{E} \subseteq 2^{V}$ ) and $\kappa: \mathcal{E} \rightarrow[n]$ be a (total) coloring of the edges. For $i \in[n]$ let

$$
\mathcal{E}_{i}=\{E \in \mathcal{E} \mid \kappa(E)=i\}
$$

be the set of those edges that have color $i$, we call $\mathcal{E}_{i}$ the $i$ th color class.

If $\mathcal{E}^{\prime} \subseteq \mathcal{E}$ and $i \in[n]$ let

$$
\lambda_{i}=\lambda_{i}\left(\mathcal{E}^{\prime}\right)=\left|\mathcal{E}^{\prime} \cap \mathcal{E}_{i}\right|,
$$

furthermore let

$$
\chi\left(\mathcal{E}^{\prime}\right)=\left(\lambda_{1}, \ldots, \lambda_{n}\right)
$$

be the profile of $\mathcal{E}^{\prime}$. The balanceness of an edge set $\mathcal{E}^{\prime} \subseteq \mathcal{E}$ w.r.t. the coloring $\kappa$ is defined by

$$
\operatorname{bal}\left(\mathcal{E}^{\prime}\right)=\operatorname{bal}_{\kappa}\left(\mathcal{E}^{\prime}\right)=\min _{i \in[n]} \lambda_{i}\left(\mathcal{E}^{\prime}\right) .
$$

$\mathcal{M} \subseteq \mathcal{E}$ is called a matching, if $E_{1}, E_{2} \in \mathcal{M}$ implies $E_{1} \cap E_{2}=\emptyset$ (in other formulation $\mathcal{M}$ is a set of independent edges). The matching balanceness of 
the hypergraph $\mathcal{H}$ w.r.t. the coloring $\kappa$ is defined by

$$
\operatorname{bal}(\mathcal{H})=\operatorname{bal}_{\kappa}(\mathcal{H})=\max _{\mathcal{M} \text { is a matching in } \mathcal{H}} \operatorname{bal}(\mathcal{M})
$$

Let $\mathcal{B}_{n, k, d}$ denote the following $k^{d}$-uniform hypergraph $(k \geq 2, d \in[n])$. The vertices of $\mathcal{B}_{n, k, d}$ are words of length $n$ over the alphabet $\Sigma=\{0, \ldots, k-$ $1\}$. The edges are those $k^{d}$-sets $E$, called $d$-spaces, that have an index set $I \subseteq[n],|I|=d$, such that for each $u=t_{1} \cdots t_{n} \in E$ and $v=t_{1}^{\prime} \cdots t_{n}^{\prime} \in E$ the property $t_{j}=t_{j}^{\prime}$ holds whenever $j \notin I$. For $k=2$ and $d=1, \mathcal{B}_{n, k, d}$ is nothing else, but the $n$-cube, $B_{n}$ (the edges are those pair of $n$-bit strings that have Hamming distance 1).

There is a natural coloring $\kappa_{\text {nat }}$ of $\mathcal{B}_{n, k, d}$ with $\left(\begin{array}{l}n \\ d\end{array}\right)$ colors, those edges are colored with the same color that have the same $I$ in the definition of the edges of $\mathcal{B}_{n, k, d}$. Each color class contains $k^{n-d}$ edges. As a special case, the edges of $B_{n}$ are colored by $n$ colors according to the $n$ parallel classes, each color class has $2^{n-1}$ edges.

Let us introduce the short notation

$$
b(n, k, d)=\operatorname{bal}_{\kappa_{\text {nat }}}\left(\mathcal{B}_{n, k, d}\right) .
$$

Given an $r$-uniform hypergraph $\mathcal{H}=\langle V, \mathcal{E}\rangle$ and coloring $\kappa: \mathcal{E} \rightarrow[n]$ we call a matching $\mathcal{M}$ a maximum balanced matching if

$$
\operatorname{bal}(\mathcal{M})=\min \left\{\min _{i \in[n]}\left|\mathcal{E}_{i}\right|,\left\lfloor\frac{|V|}{r n}\right\rfloor\right\}
$$

holds. The balanceness of a matching obviously can not be larger than the RHS of (9). For the case of $B_{n}$, this RHS is equal to $\left\lfloor 2^{n} / 2 n\right\rfloor$. So, our main result, Theorem 1.1, can be formulated in the following way.

$$
b(n, 2,1)=\left\lfloor 2^{n} / 2 n\right\rfloor \quad(n \neq 2) .
$$

\section{Balanced $d$-spaces}

In this section we prove a general lower bound on $b(n . k, d)$. Note, that this lower bound is an initial result, determining the exact value remains open in most of the cases. 
Lemma 5.1. Let $\mathcal{S}$ be the multiset, that contain exactly $s$ copies of each element of $\left(\begin{array}{c}{[n]} \\ d\end{array}\right)$, where $s=d / \operatorname{gcd}(d, n-d+1)$. Then for the multiset $\mathcal{T}$ of $(n-d+1) s / d$ copies of $\left(\begin{array}{c}{[n]} \\ d-1\end{array}\right)$, there exists a bijection $\varphi: \mathcal{S} \rightarrow \mathcal{T}$, such that $S \supset \varphi(S)$ holds for all $S \in \mathcal{S}$.

Proof. The bipartite graph $\langle\mathcal{S}, \mathcal{T}, \mathcal{E}\rangle$, where $\{S, T\} \in \mathcal{E} \Leftrightarrow T \subset S$ is $(n-d+1) s$-regular, therefore it has a matching.

Corollary 5.1. Given $s\left(\begin{array}{l}n \\ d\end{array}\right)$ edges (d-spaces) of $\mathcal{B}_{n, k, d}$, where $s=d / \operatorname{gcd}(d, n-$ $d+1)$ and exactly $s$ of the edges have the same color in $\kappa_{\text {nat }}$ for each color class. Then we can replace each $d$-space by $k(d-1)$-spaces of the same color class of $\mathcal{B}_{n, k, d-1}$ in such a way, that there will be exactly $k(n-d+1) s / d$ edges in each of the $\left(\begin{array}{c}n \\ d-1\end{array}\right)$ color classes of $\mathcal{B}_{n, k, d-1}$ w.r.t $\kappa_{\text {nat }}$.

Proof. Let $\mathcal{S}$ correspond to the color classes of $\mathcal{B}_{n, k, d}$, while $\mathcal{T}$ to the color classes of $\mathcal{B}_{n, k, d-1}$. Replace a $d$-space of color class $S \in \mathcal{S}$ by $k(d-1)$-spaces of the color class $\varphi(S)$.

The following theorem gives a recursive method to count a general lower bound for $b(n, k, d)$.

\section{Theorem 5.1.}

$$
b(n+1, k, d) \geq k b(n, k, d)-k s\left\lceil\frac{d b(n, k, d)}{(n+1) s}\right\rceil,
$$

where $s=d / \operatorname{gcd}(d, n-d+1)$.

Proof. Suppose, that we have a matching $\mathcal{M}_{n}$ having $b(n, k, d) d$-spaces of each color. $V\left(\mathcal{B}_{n+1, k, d}\right)=X_{0} \cup \cdots \cup X_{k-1}$, where $X_{i}=\left\{u i \mid u \in V\left(\mathcal{B}_{n, k, d}\right)\right\}$ $(0 \leq i \leq k-1)$. Let the edge set $\mathcal{D}$ consist of $k$ isomorphic copies of $\mathcal{M}_{n}$ on the vertex sets $X_{i}(0 \leq i \leq k-1)$. $\mathcal{D}$ have a profile vector

$$
\chi(\mathcal{D})=(k b(n, k, d), \ldots, k b(n, k, d), 0, \ldots, 0),
$$

where we have 0 for those $d$-sets of $[n+1]$, that contain $n+1$ (let these be the last $\left(\begin{array}{c}n \\ d-1\end{array}\right)$ components).

Replace $s d$-spaces of each color by $(d-1)$-spaces over $X_{0}$ according to Corollary 5.1. Each type of $(d-1)$-space will occur $k(n-d+1) s / d$ times. Do exactly the same for $X_{1}, \ldots X_{k-1}$. Replace each $k$ corresponding $(d-1)$-spaces 
in $X_{0}, \ldots, X_{k-1}$ by a single $d$-space. So the first $\left(\begin{array}{l}n \\ d\end{array}\right)$ components of $\chi(\mathcal{D})$ are decreased by $k s$, while the last $\left(\begin{array}{c}n \\ d-1\end{array}\right)$ one are increased by $k(n-d+1) s / d$.

Repeating this transformation $\ell$ times, we have the following profile for the actual edge set $\mathcal{D}$.

$\chi(\mathcal{D})=\left(k b(n, k, d)-\ell k s, \ldots, k b(n, k, d)-\ell k s, \ell k \frac{(n-d+1) s}{d}, \ldots, \ell k \frac{(n-d+1) s}{d}\right)$.

Let $\ell_{0}$ be the least integer satisfying

$$
k b(n, k, d)-\ell_{0} k s \leq \ell_{0} k \frac{(n-d+1) s}{d},
$$

i.e., $\ell_{0}=\lceil d b(n, k, d) /(n+1) s\rceil$. Then all components of $\chi(\mathcal{D})$ is at least the RHS of (10).

We omit the elementary, but space and paper consuming counting of the following.

Corollary 5.2. Let $n_{0} \geq k d /(k-1)$. Then we have

$$
\left\lfloor\frac{k^{n-d}}{\left(\begin{array}{l}
n \\
d
\end{array}\right)}\right\rfloor \geq b(n, k, d) \geq \frac{k^{n-d}}{\left(\begin{array}{l}
n \\
d
\end{array}\right)} \frac{\left(\begin{array}{c}
n_{0} \\
d
\end{array}\right)}{k^{n_{0}-d}}\left(b\left(n_{0}, k, d\right)-d \frac{n_{0}+1}{n_{0}-d+1} \frac{\left(n_{0}+2-d\right) k}{\left(n_{0}+2-d\right) k-n_{0}-2}\right) .
$$

We can see, that there is a big room to improve. For $d=1$ the same inductive argument gives somewhat better.

Theorem 5.2. For $n \geq 4$

$$
\left\lfloor\frac{k^{n-1}}{n}\right\rfloor \geq b(n, k, 1) \geq \llbracket \frac{k^{n-1}}{n} \rrbracket_{k} .
$$

Proof. There is a maximum balanced matching for $n=4$. Suppose, that we have a matching $\mathcal{M}_{n}(n \geq 4)$ having $\llbracket k^{n-1} / n \rrbracket_{k} 1$-spaces in each direction. Let $V\left(\mathcal{B}_{n+1, k, 1}\right)=X_{0} \cup \cdots \cup X_{k-1}$, where $X_{i}=\left\{u i \mid u \in V\left(\mathcal{B}_{n, k, 1}\right)\right\}(0 \leq i \leq k-1)$. Take isomorphic copies $\mathcal{M}_{n}^{(i)}$ of $\mathcal{M}_{n}$ in each $X_{i}$ and add the 1-spaces that consist of the corresponding vertices of $V\left(\mathcal{M}_{n}^{(i)}\right)-X_{i}(0 \leq i \leq k-1)$.

A set of $k 1$-spaces of direction $r, E_{i}=\left\{t_{1} \cdots t_{r-1} x t_{r+1} \cdots t_{n} i \mid 0 \leq x \leq\right.$ $k-1\}(0 \leq i \leq k-1)$ can be replaced by another $k 1$-spaces of direction $n+1, E_{i}^{\prime}=\left\{t_{1} \cdots t_{r-1} i t_{r+1} \cdots t_{n} x \mid 0 \leq x \leq k-1\right\}(0 \leq i \leq k-1)$. Consider 
again the following transformation: replace $k n$ edges, $k$ from each direction and of the above type, by $k n$ edges of direction $n+1$.

Repeat the transformation while the number of edges of direction $i(i \in$ $[n])$ is bigger than $\left\lfloor k^{n} /(n+1)\right\rfloor$. Note, that the initial number of edges of direction $i(i \in[n])$ in $\mathcal{B}_{n+1, k, 1}$ is divisible by $k$. The transformations do not change this property, so the statement follows.

\section{References}

[1] C. D. Savage. A Survey of Combinatorial Gray Codes. SIAM Review 39 (4): 605-629, 1997.

[2] G. O. H. Katona and K. Tichler. Search when the lie depends on the target, submitted.

[3] F. Gray. Pulse Code Communication, U.S Patent No. 2632058, March 1953.

[4] G. S. Bhat and C. D. Savage. Balanced Gray codes. Electronic Journal of Combinatorics 3 (1): R25, 1996.

[5] J. Robinson and M. Cohn. Counting Sequences. IEEE Transactions on Computers, C-30: 17-23, 1981. 